\title{
1A Nationwide Analysis of U.S. Racial/Ethnic Disparities in Emergency Department Patients with Mental Health and Substance Use Disorders
}

Joanne Weinreb ( $\sim$ jweinreb@citytech.cuny.edu )

New York City College of Technology https://orcid.org/0000-0002-6545-5917

Penina Gavrilova

New York City College of Technology

Jonathan Avery

Weill Cornell Medicine

Sean M. Murphy

Weill Cornell Medicine

Jyotishman Pathak

Weill Cornell Medicine

\section{Research Article}

Keywords: Emergency department, Healthcare disparity, Mental health and substance use disorders, Time to treatment

Posted Date: September 16th, 2021

DOI: https://doi.org/10.21203/rs.3.rs-892560/v1

License: (1) This work is licensed under a Creative Commons Attribution 4.0 International License. Read Full License 


\title{
A Nationwide Analysis of U.S. Racial/Ethnic Disparities in Emergency Department Patients with Mental Health and Substance Use Disorders
}

\author{
Joanne Weinreb, PhD ${ }^{1 *}$, Penina Gavrilova, BS ${ }^{1}$, Jonathan Avery, MD ${ }^{1}$ Sean M. Murphy, \\ $\mathbf{P h D}^{2}$, Jyotishman Pathak, $\mathbf{P h D}^{2}$ \\ ${ }^{1}$ New York City College of Technology, Brooklyn, New York.; ${ }^{2}$ Weill Cornell Medicine, \\ New York, New York.
}

*Corresponding author - email: jweinreb@ citytech.cuny.edu

\begin{abstract}
Background: Racial and ethnic health disparities have been linked with inequalities in access to health care and outcomes. The present study considers whether inequalities persist between racial/ethnic groups among patients with mental health or substance use disorders who visit the emergency department (ED).

Methods: We analyzed data from the National Hospital Ambulatory Medical Care Survey (NHAMCS) from 20122018, assessing health disparities among patients diagnosed with mental health or substance use disorders by observing whether significant differences exist in ED wait time and length of visit (LOV) for patients of different races/ethnicities. Stratified models were performed to further understand the impact of regions across the U.S., year, and triage level on the association analysis.

Results: From 2012-2018, non-Hispanic Black and Hispanic patients experienced significantly longer ED wait times and LOV as compared to White patients. Patients with private insurance experienced significantly shorter wait times compared to patients with self-pay, and shorter LOV than those with Medicaid/ Children's Health Insurance Program, or Medicare. Male patients had significantly longer LOV compared to female patients. We observed year by year differences in wait times of non-Hispanic Black patients with improvement appearing between the years 2013 to 2016, while LOV remained consistently longer. We observed both regional and triage level differences, with the U.S. Northeast presenting with the most disparities. Additionally, we noted a general upward trend of substance use disorder (SUD) diagnoses.

Conclusion: Our analysis suggests that while there has been an overall improvement in median ED wait time through the years, non-Hispanic Black and Hispanic patients experience significantly longer ED wait time compared to non-Hispanic White patients. Additionally, non-Hispanic Black and Hispanic patients have a significantly longer ED LOV compared to non-Hispanic White patients.
\end{abstract}

\section{Keywords}

Emergency department, Healthcare disparity, Mental health and substance use disorders, Time to treatment. 


\section{Background}

Health disparities, the inequality between patients of different groups, are seen to affect patients in several ways, including access to health care, quality of care, delays in getting seen by medical professionals, and delays in medical treatment (1). Groups of patients experiencing health inequalities could be marginalized based on their age, gender, race, ethnicity, sexual orientation, geography, socio-economic status, or other socio-demographic characteristics (2).

Health disparities are highly related to the effect that social inequalities have on population health $(3,4)$. The idea behind population health is to improve the overall health of a community and reduce health disparities $(5,6)$. Racial and ethnic inequities are one of the most common sources of disparities (7). As the U.S. population becomes more diverse (8), the issue of racial and ethnic health disparities becomes an increasingly important public health policy consideration.

Racial and ethnic health disparities are observed across all aspects of healthcare, including emergency departments (EDs). Disparity among ED patients can be seen in their wait time to see an ED provider and the ED length of visit (LOV) $(9,10)$. These two categories report on the timeliness of services provided to the patient. Patients with acute issues must be seen in a timely manner as delays can potentially affect timely ED triage, diagnosis, treatment, and patient outcomes $(10,11)$. Additionally, it can impact a patients' experience as a visit to the ED is, in general, an individual's first experience with the healthcare system and can dramatically impact their overall health timeline going forward with a negative experience (12).

Patients with either mental health or substance use (MH/SU) disorders represent an increasing percentage of all ED visits (13). A previous study reported an increase from 5.4\% of total ED visits in 2000 to $12.5 \%$ in 2007 (13). From 2006 to 2011 ED visits for substance-related disorders increased by $48 \%$ and alcohol-related disorders increased by $34 \%$ as compared to an overall increase of $4.5 \%$ for all ED visits (14).

Increasing use of the ED by MH/SU patients has been identified as a contributor to general ED overcrowding (15). EDs have difficulty in finding psychiatric inpatient beds for their MH/SU patients, leading to elongated boarding, which in turn leads to fewer beds available for other ED patients (15). MH/SU patients may use the ED to treat complications from their disorders when access to psychiatric services is limited (16). Additionally, there is evidence that $\mathrm{MH} / \mathrm{SU}$ represent a large portion of repeat visits to the $\mathrm{ED}(17,18)$.

Studies focused on specific MH/SU disorders have highlighted issues in the ED. Borderline personality disorder patients were shown to be both frequent and repeat visitors to the ED. Negative and sometimes volatile interactions have occurred between these patients and the ED staff who may not be well trained to treat these disorders. Additionally, some health professionals have a negative attitude towards working with individuals with an SUD $(19,20)$. Opioid use disorder in 2018 affected 2 million people (21). From 2010-2015 the rate of opioid deaths amongst Black communities rose by $43 \%$ (22). This increase is largely a function of heroin and synthetic opioids (23) due, in part, to lower prescribing rates for prescription opioids (24), drug price differences and cultural norms (22).

Our work builds on previous analyses of health disparities in the population of ED patients using LOV and wait time as measurements(25). Analysis of data from 2003-2005 indicated that non-Hispanic Black patients had significantly longer ED LOVs before being admitted to the hospital, compared to non-Hispanic White patients (10). Hispanic ED patients experiencing acute gastric illness between 1997 and 2006 were shown to have longer wait time compared to non-Hispanic White patients (26). MH/SU ED adult patient data from 2009-2011 were analyzed, with findings indicating longer wait times for non-Hispanic Black patients, compared to non-Hispanic White patients. The model also examined differences based on insurance type or metropolitan statistical area status, although no significant differences were observed (27).

The primary purpose of our study is to examine health disparities in ED patients with $\mathrm{MH} / \mathrm{SU}$ disorders using a nationally-representative dataset covering the years 2012-2018. Our analysis includes MH/SU patients across all age groups and U.S. regions. Specifically, this study investigates ED wait times and length of visits as indicators of disparities. Additionally, we looked more closely at how factors of time (year by year), location (region), and 
severity of illness (triage level) impact a patient's ED experience. The findings from our study could be used to inform ED-related policy decisions at all levels.

\section{Methods}

\section{Study design and setting}

We performed a retrospective analysis of the U.S. National Hospital Ambulatory Medical Care Survey (NHAMCS) data from 2012-2018. Maintained by the U.S. Centers for Disease Control and Prevention (CDC), NHAMCS is based on a nationwide sample of all visits for ambulatory care services in hospital emergency and outpatient departments (outpatient department data are not available from 2012-2018), including hospital-based ambulatory surgery centers, from non-institutional general and short-stay hospitals, while excluding federal, military and Veterans Health Administration hospitals. Data collected by the survey include patient demographics, admission and discharge details, insurance type, and clinical information across all regions in the U.S. (all 50 states and the District of Columbia). The details of the NHAMCS methods and procedures are described in the NHAMCS data file (NAMCS/NHAMCS - About the Ambulatory Health Care Surveys, 2019).

\section{Selection of Participants}

Across the seven years of our study, NHAMCS logged 155,602 records, a representative sample of 954,128,804 visits. We identified all patients who had an NHAMCS primary, secondary, or tertiary diagnosis of a mental health or substance use disorder, matching the International Classification of Diseases, Ninth Revision, Clinical Modification (ICD-9-CM) codes for data from 2012-2015 and ICD-10-CM codes for 2016-2018. These included mental health and substance use disorders (ICD-9-CM:290.0-319.0, ICD-10-CM F-10-F99), and cause of injury for self-inflicted injury or suicide attempt (ICD-9-CM:E950.0-E959.9, ICD-10-CM:X71-X83) or the NHAMCS indicator for self-inflicted intentional injuries. Also included were patients who were transferred to a psychiatric hospital, or were admitted into mental health or SUD detoxification units. We excluded all patients who were dead on arrival, and those who left before being triaged. Additionally, all visits with blank or not applicable values in wait time and race/ethnicity categories were removed from the analysis. LOV data were not released by the National Centers for Health Statistics due to data quality issues for the years 2016 and 2017; these years were excluded from all LOV calculations.

\section{Outcome measures and covariates}

The primary outcome measures were participant ED wait time and LOV. Wait time is the calculated number of minutes between ED arrival and the point they were seen by a provider. LOV is calculated as the difference in minutes between the ED arrival and departure times, and includes wait time, mental health response and evaluation, treatment, and the wait for discharge. Both metrics have been used in other studies $(10,13,27-29)$, and are often reported as quality measures for emergency care (30). The other variables of interest for this study included gender; race; ethnicity; diagnosis; patient visit weight; triage level; insurance type; region; and the specific $\mathrm{MH} / \mathrm{SU}$ diagnosis (8).

Patient and hospital level covariates were obtained including patient demographics, diagnosis, patient visit weight; triage level; insurance type; and region (8). Race and ethnicity were taken from a single variable with missing data imputed by NHAMCS using a model-based single, sequential regression method which included the variables including: Census variables for ZIP code level race and ethnicity population estimates, patient age, sex, race, and ethnicity; triage level; log of wait time; 3-digit ICD-9/10-CM codes for primary diagnosis; who completed the Patient Record form; year of visit; type of emergency service area. Race and ethnicity has four possible values: nonHispanic White, non-Hispanic Black, Hispanic, and non-Hispanic other.

Triage level describes the immediacy with which a patient should be seen: immediate ( 0 minutes), emergent (1-14 minutes), urgent (15-60 minutes), semi-urgent (61-120 minutes), nonurgent (121 minutes to 24 hours), and unknown or no triage. No mention of an immediacy rating or triage level in the medical record, indicates that the hospital did not perform triage, or the patient was dead on arrival. Insurance type was categorized as Medicaid or Children's Health Insurance Program (CHIP), Medicare, private insurance, worker's compensation, other (Tricare, private charitable organizations, state or local governments, and other liability insurances), self-pay, no charge/charity, and 
unknown. As the percentages of patients for worker's compensation (<1\%), no charge/charity (1\%), and other (5\%) were low, and unknown (9\%) is non-specific, we rolled these into a separate category called "Other or unknown" for our analysis. A hospital was identified as being located in one of four regions: Northeast, Midwest, South and West.

\begin{abstract}
Analysis
All data analysis was performed using $\mathrm{R}$ version 3.5. For each variable and descriptive statistic category medians and one-way ANOVAs were performed.. We applied the provided weights, such that our results are nationally representative. Multivariable linear regression models were performed for two dependent variables, wait time and LOV, and included independent variables such as gender, race/ethnicity, insurance type, and region. Logtransformation was performed on both the dependent variables to approximate a normal distribution as normality tests indicated that our data did not adhere to a Gaussian distribution. Statistical significance is assessed at p-value < 0.01 as recommended by NHAMCS to decrease the possibility of Type 1 errors. Our first models looked exclusively at the patient level covariates gender, race/ethnicity, and insurance type. Our second model incorporated the hospital level covariate of region. In addition, all models were further stratified by year, hospital region, and recorded triage levels.
\end{abstract}

Included as supplemental tables and figures are a deeper analysis of the specific $\mathrm{MH} / \mathrm{SU}$ diagnosis that were included within our study. These analysis reflect the change in prevalence of diagnosis over time and race/ethnicity.

\title{
Results
}

\section{Description of the populations}

There were a total of 12,996 unique (74,316,000 weighted) patients with a diagnosis for a MH/SU disorder admitted to the ED between 2012-2018. MH/SU represents $8 \%$ of all ED visits during this time frame. Using weighted estimates, the number of MH/SU ED visits increased by $60 \%$ from $2012(n=7,560,000)$ to $2018(n=12,083,583)$ with a spike in $2016(n=13,898,000)$, while the number of general ED visits remained similar (2012: $n=130,869,572$; 2018: $n=129,973,941)$. Patients with MH/SU disorder experienced a 37\% (212 vs. 155 minutes) higher LOV than the general ED population as a median (excludes 2016-2017 when LOV was not available). The longest median wait time was in 2013 (24 minutes), and the highest LOV was in 2018 (231 minutes) (Table:1).

Participating hospitals were identified as being located in one of four regions: South, Midwest, Northeast and West. The Southern region experienced the highest level of MH/SU ED visits (30\%), while the Northeast region experienced the fewest (17\%). The Northeast region experienced significantly ( $\mathrm{p}<.001)$ longer wait times $(26$ minutes) and LOV (227.5 minutes) as compared to all other regions. The Midwest had the lowest median wait time (17 minutes) and one of the lowest LOV (190 minutes) (Table:1).

The majority of patients were male $(n=37,954,000,51 \%)$, non-Hispanic Whites $(n=46,938,000,63 \%)$, and were insured by Medicaid or CHIP ( $n=25,029,000,34 \%)$. Ages ranged from 0 to over 94 with a median of $37.46 \%$ of triaged patients were identified as urgent. Both genders had similar median wait times (19 minutes), but males (226 minutes for males; 198 minutes for females) exhibited significantly longer LOV ( $p<.001)$. Non-Hispanic Black patients experienced the longest wait times and LOV (22 and 248 minutes, respectively) and non-Hispanic White had the lowest rates. Females' median LOV was 7\% longer than males' when looking at all ED visits, while males had a $14 \%$ larger median LOV when focused on our MH/SU subset. Additionally, the male MH/SU ED patients are in the majority (51\%), while in the general ED population females represent $57 \%$ of the population.

Detailed analysis of the specific diagnosis are included as two tables in the supplement. Supplemental Table:1 looks at the racial breakdown across all MH/SU codes, and Supplemental Table:2 reports on SUD codes specifically. The most frequent diagnoses were psychoactive substance use $(35 \% ; n=39,430,863)$; followed by anxiety and other nonpsychotic mental disorders $(18 \% ; \mathrm{n}=20,259,601)$. Additionally, $11 \%(\mathrm{n}=12,639,012)$ were identified as intentional self-harm, and $9 \%(\mathrm{n}=10,421,311)$ were either transferred to a psychiatric hospital or admitted to a mental health or a detox unit. Alcohol use disorder was the most $(38 \% ; n=13,361,025)$ observed SUD code followed by tobacco/nicotine $(31 \% ; 5,149,973)$, while opioids $(5 \% ; \mathrm{n}=1,904,609)$, cocaine $(3 \% ; \mathrm{n}=1,022,330)$, and cannabis $(3 \% ; n=1129341)$ were observed much less frequently. 
Non-Hispanic Whites made up the largest percentage of most diagnosis categories, as would be expected, since they were $60 \%$ of the total $\mathrm{MH} / \mathrm{SU}$ study population. Of note, is that non-Hispanic Blacks receive more schizophrenia and other non-mood psychotic diagnoses $(36 \% ; n=2,137,910)$ and fewer mental disorders due to know physiological conditions $(14 \% ; n=517557)$ than what would be expected as they are $24 \%$ of the general study population. Of the observed SUD codes, non-Hispanic Blacks had fewer opioid $(11 \% ; \mathrm{n}=218,807)$ and amphetamine and other stimulants $(12 \% ; \mathrm{n}=157,859)$ diagnoses, while being overrepresented for cocaine and cannabis $(53 \% ; \mathrm{n}=540,747$, $27 \% \mathrm{n}=305,010$, respectively), compared to the general MH/SU population (non-Hispanic Blacks $24 \%$ ). While Hispanics, who represent $14 \%$ of the study population, had higher rates of amphetamine and other stimulant (24\%; $\mathrm{n}=308308)$ and cannabis $(22 \% ; \mathrm{n}=247114)$ diagnoses. Non-Hispanic White patients have much lower rates of cocaine and cannabis $(29 \% ; \mathrm{n}=301024,49 \% ; \mathrm{n}=552181$, respectively) as compared to the expected rate $(60 \%)$. Supplemental Figure 1 displays the yearly trends for each diagnosis. Looking across all seven years we see a rise in cannabis use disorder across all ethnicities. Cocaine use disorder nearly tripled amongst Hispanics, and increased by 50\% amongst non-Hispanic Blacks. Opioid use disorder rose both within the non-Hispanic White and non-Hispanic Black populations, with non-Hispanic Blacks accounting for larger percentage of the cases (7\%(2012) - 16\%(2018) (Supplemental Figure:2).

\section{Multivariable results}

The multivariable linear regression model from 2012-2018 using only patient covariates showed that non-Hispanic Black and Hispanic patients experienced significantly longer ED wait times relative to non-Hispanic White patients. Patients with self-pay experience a significantly longer wait time than patients with private insurance. Non-Hispanic Black and Hispanic patients experience significantly longer LOV compared to non-Hispanic White patients. Individuals with Medicaid or CHIP, Medicare, and unknown or other experience significantly longer LOVs compared to those with Private (Commercial) Insurance. Significantly longer LOVs were also seen for male patients compared to female patients. (Table:2)

Hospital level dependent variable region was added to the second multivariable linear regression model. While nonHispanic Black patients continue to show significantly longer wait times ( $<<.001)$, Hispanic patients' wait time differences are insignificant. Using the U.S. South as the reference region, since that was the largest percentage of the population, patients in the U.S. Northeast $(\mathrm{p}<.0001)$ showed both significantly longer wait times and LOV while patients in the U.S. West ( $\mathrm{p}<.0001)$ only showed longer LOV.

\section{Region by Region Analysis}

The Southern region had the most MH/SU ED visits (34\%) (Table:1). Non-Hispanic White patients are the greatest percentages across all regions, with the Midwest having the highest percentage (68.6\%). The percentage of nonHispanic Blacks is the highest in the South (26.1\%) (Figure:1a). Across all four regions the rates of non-Hispanic Black patients' usage of the ED, both total and $\mathrm{MH} / \mathrm{SU}$ patients are far higher than their representation within the population (Supplemental Table:3). Non-Hispanic White patients have the greatest range in wait times across all regions. Non-Hispanic Black patients in the Northeast region exhibit a distribution skewed towards higher wait times (Figure:1c) and longer LOVs in all regions except the West (Figure:1d).

For the region stratified multivariable linear regression model using patient level covariates, only the Northeast region showed significantly longer ED wait times for non-Hispanic Black and Hispanic patients relative to nonHispanic White patients. Compared to patients with private insurance, those who self-pay experience a significantly longer wait time $(\mathrm{p}<0.01)$ in the South, while those with Medicaid have a longer wait in the Northeast and Medicare patients wait longer in the West. Non-Hispanic Black patients experience significantly longer LOV compared to non-Hispanic White patients $(\mathrm{p}<0.001)$ in all regions, and Hispanics in the Northeast show a similar disparity. Individuals with Medicare experience significantly longer LOVs ( $\mathrm{p}<0.001)$ compared to those with Private insurance in the South and the North. The Northeast stands out as the region with the most health disparity when analyzing both wait times and LOV as well as comparing insurance types (Table:3).

\section{Year by Year Analysis}

Results show a 18\% decline in median ED wait time from 2012 (22 minutes) to 18 minutes in 2018, while dropping to 15 minutes in 2016 (Table:1). In 2012, non-Hispanic Black patients experienced the highest median wait time of 31 with $25 \%$ and $75 \%$ quartiles of 14 and 71, respectively. Non-Hispanic White, Hispanic, and non-Hispanic other patients had medians of 20. In 2013 non-Hispanic Black, Hispanic, and non-Hispanic other patients reported wait 
times between 25-27, while the non-Hispanic White population had waited only 22 minutes. (Table:1) Interquartile ranges are generally wider for non-Hispanic Black and Hispanic, with the exception of 2015 for the Hispanic ethnicity. (Figure:2) The greatest outlier was from a 2016 non-Hispanic Black patient who waited 1390 minutes. LOV quartiles show that while the median for non-Hispanic Black patients shrank 14\% over four years from 20122015 they still experienced the longest visits. In 2016 and 2017 NHAMCS did not record LOV data. Looking at the data from 2018 (Figure:2) interquartile ranges for all racial groups were much larger, and the median LOV for all groups in comparison to non-Hispanic White was elevated. The median LOV for Hispanic patients was comparatively larger in 2013, 2015 and 2018. The longest LOVs were over 5500 minutes, close to 4 days.

The MH/SU racial population for each year closely aligns with racial breakdown for all ED visits (Supplemental Figure:3b). In 2013 and 2016 the percentage of non-Hispanic White patients who visited the ED increased, while non-Hispanic Black and Hispanic patients decreased slightly. When looking at the payment source for each year (Supplemental Figure:3c), one can see that while the number of patients who paid using Medicaid increased from $26 \%$ in 2012 to $41 \%$ in 2018 , the number of self-pays decreased from $17 \%$ to $9 \%$.

A multivariable linear regression model using patient covariates stratified by year showed that while in 2012 nonHispanic Black patients still showed a significantly longer wait time, for the subsequent years the significance steadily declined until 2017 and 2018 when the significant disparities were seen again (Table:4). LOV persistently showed a significant increase for non-Hispanic Black patients with improvement only seen in 2015. Hispanics showed increased LOV for 2013, 2015 and 2018. Insurance types were a significant indicator for LOV only in 2012 and 2013.

\section{Triage Level Analysis}

Triage level was known for 9,716 (75\%) unique ED visits $(55,234,810$ weighted $74 \%)$ for $\mathrm{MH} / \mathrm{SU}$ patients. Patients triaged as immediate were less than $1 \%$ of the population, and urgent was the largest (34\%) group. Median wait time varied from 12 minutes for immediate triage level to 20 minutes for urgent and semi-urgent. Patients triaged as nonurgent experienced the shortest median LOVs (118 minutes), while emergency patients exhibited the highest median LOV (309 minutes) (Table:3). The longest wait time (1140 minutes) was for a non-urgent patient, and the longest LOV (5560 minutes) was incurred by an emergency patient.(Figure:3).

For the multivariable linear regression model using patient covariates stratified by triage level there were no reported disparities for those patients triaged as immediate. At a significance of .01 no disparities in wait time are reported, if the significance is relaxed to .05 then we see longer wait times for non-Hispanic Black patients at the emergent and urgent triage levels and longer times for non-urgent Hispanic patients. Non-Hispanic Black patients experience longer LOV at all levels except immediate. Hispanic patients display significantly longer LOV when triaged as both urgent and semi-urgent. Urgent and semi-urgent patients showed significantly longer LOV for patients paying with Medicare. (Table:5)

\section{Discussion}

This study examined health disparities in ED patients with MH/SU disorders using the NHAMCS dataset from 2012-2018. MH/SU represent a growing percentage of total ED visits. While ED admissions of $\mathrm{MH} / \mathrm{SU}$ patients increased 60\% from 2012 to 2018 the general ED population was fairly stable. This shift may be partially due to a shortage of mental health providers (31). There is a decreasing number of residencies in psychiatry, unbalanced geographic distribution of providers, and problems with recruitment (32). Additionally, some of this increase may be accounted for by the ongoing opioid crisis (21).

Over the seven years of the study, we noted a $22 \%$ reduction in median ED wait time for MH/SU patients. Numerous efforts have been put in place to improve overall ED wait times and LOV. These efforts include improvements in ED patient triaging, including increased use of telemedicine in EDs, and co-location of general practitioner clinics. Studies show that triage performed by physicians, instead of the common method of nurses 
conducting triage, speeds up the patient flow and reduces ED overcrowding, by initiating patient assessment and testing earlier in the process (33). Telemedicine evaluation of ED patients, specifically low-acuity patients, may reduce both wait time and LOV as these patients are treated remotely by appropriate specialists (34). Having colocation of general practitioner services is convenient for patients experiencing non-urgent problems. It was shown that co-location of services reduced wait time on average 19\% (33). Additionally, some EDs have implemented Point-of-Care Testing (POCT) which allows for testing to be done outside of the hospital's central laboratory and near patient's bedside, which speeds up results (35). Several studies show improvement in patient outcomes and a decrease in the LOV after POCT implementation $(33,35)$. EDs have established case management programs which reduced LOV by $39 \%$ (36).

However, despite these improvements, health disparities for the non-Hispanic Black and Hispanic patient populations can be seen in their experiencing significantly longer wait times and LOV during ED encounters as compared to non-Hispanic Whites. These delays can affect both the patients' ED perceived experience and their health outcomes by causing delayed treatment and increased chances of medical errors (13).

Health disparities were also apparent when looking at the payment source for the ED visit. Patients who self-pay for their ED visits are approximately equally distributed across the races and experience longer wait times as compared to those covered by private insurance (Supplemental Figure:3d). Additionally, patients with private insurance experience shorter LOVs in the ED. Extensive wait times for uninsured or low-income patients result in patients leaving the ED without seeing a physician or leaving the ED against medical advice $(37,38)$. Factors that result in extended LOV for self-pay and uninsured patients include the struggle of faster boarding (admitted to inpatient or outpatient facility) (13). Prolonged boarding for these insurance types is due in part to difficulty locating an appropriate transfer facility due in part to scarcity of this resource.

We noted disparities in the MH/SU population based on gender. Male MH/SU ED patients consistently experienced significantly longer LOV than females. This differs from the general ED population where females exhibit longer mean LOVs (Karaca et al., 2012). One possible explanation for this is that male MH/SU patients that present themselves in the ED have more comorbid mental health and substance use disorders like alcohol, cocaine and suicide and are therefore more likely to have complications leading to longer ED visits.

Across all four regions the rates of non-Hispanic Black usage of ED, both total and MH/SU patients, are far higher than their representation within the population. Non-Hispanic Black patients use the ED for psychiatric services at a much higher rate than other racial groups. This does not correlate to higher incidences of psychiatric need by the Black community in general (39). High ED usage for mental health is an indication of a health system that is functioning less optimally. EDs are considered a doorway to mental health hospitalization and involuntary commitment, and non-Hispanic Black patients are over-represented in that arena (39). An increased rate of unmet needs for mental healthcare frequently lead to increased boarding times in EDs which would increase the LOV $(13,40)$.

Non-Hispanic Black and Hispanic are more likely to perceive discrimination in $\mathrm{MH} / \mathrm{SU}$ care incidences which is associated with early termination of treatment and may lead to degraded MH outcomes. This in turn may be a cause of their disproportionate use of ED psychiatric services as their treatment gets delayed and it becomes an emergency situation (41).

Non-Hispanic Black and Hispanic patients tend to see different physicians and in different hospitals. This may be informed and confounded by hospital access. Non-Hispanic Black and Hispanic patients, particularly those with public or no insurance, use hospitals that have longer wait times and worse access to behavioral health. These findings my be part of structural racism that drives these Black and Hispanic patients to worse, more crowded, and less resourced hospitals.

Health disparities were experienced by both non-Hispanic Black and Hispanic patients across all triage levels except for immediate as seen by longer LOVs. Triage level assignment is not standard across institutions, and can vary between practitioners even within the same department. This may indicate that triage decisions are influenced by factors other than a standardized assessment tool. Subjective factors, or providers' implicit biases may influence the decision process. Patients exhibiting the same severity of symptoms may be classified differently leading to a delay 
in necessary immediacy of care (42). Additionally, it has been reported that ED overcrowding affects the quality of triage by prolonging the wait time until triage and then potentially under-classification of the patients and missing triage scores which then may affect the patient outcomes (38).

In our analysis in year over year trends it looks like a tale told about two time periods with 2016 appearing to be a transition year. It went from being the best of improvements between 2012 to 2015 with obvious racial health disparites all but disappearing, to the worst of setbacks from 2016 to 2018 . The initial improvement may be due, in part, to the increase in the percentage of patients covered by Medicaid or CHIP, and the corresponding decrease in self-pay and unknown or other for the same period. This shift coincides with increasing state participation in the Medicaid expansion, as part of the Affordable Care Act (ACA) (29,38). In 2016 there were two noted transitions. ICD-10-CM coding was implemented, although more investigation would need to happen to identify why this would highlight racial health disparities. 2016 also heralded a change in political leadership which may have impacted the enrollment in health insurance plans offered through the ACA. We see this in our analysis when looking at payment sources by year (Supplemental Figure:3c) usage of Medicaid/CHIP grew steadily until 2016 and then in 2017 and 2018 those numbers started coming down.

Across the seven years of our study we note a general upward trend of SUD diagnoses. Cannabis rates increased across all ethnicities, which corresponds with the changing legal status of marijuana. Non-Hispanic Whites had the

largest increase opioid usage. Non-Hispanic Black patients represented a growing percent of all MH/SU patients for both cocaine and opioid usage. Some of these changes may be the result of an increased attention to these diagnoses given the opioid epidemic. Other studies have noted increased rates of opioid deaths amongst the Black communities, as well as negative attitudes that some health professionals have towards working with substance users. The Commission on Combating Drug Addiction and the Opioid Crisis final report in 2017 noted that the majority of people of color with opioid use disorder are in the lowest income bracket and are covered by public insurance which limits their access to outpatient substance use facilities (22). While these points do not explain the health disparities noted by an increase in wait time and LOV, perhaps they can help focus on areas of further study.

\section{Limitations}

There were notable limitations in the size and detail of the NHAMCS dataset. Racial and ethnic disparities often dissipate upon control of the patient's socio-economic status (29). We attempted to account for this by using insurance status, although we recognize the limitation of using insurance as a proxy for socio-economic status. We were unable to determine whether comorbidities may complicate patients' visits and would most inform LOV. Additionally, due to the large sample size, statistical significance and time trends may be common and should be confirmed by additional datasets.

To study healthcare disparities in general, it is important to study the impact of the location where services were offered. In this study, we were only able to estimate this based on the region in the country which is not granular. There is an additional variable, metropolitan statistical area, which identifies highly populated regions; however, these data were not available for 2012, and hence we did not include metropolitan statistical areas in our analysis.

Both wait time and LOV are calculated based on arrival time. Arrival time is often recorded as the time of triage, however the wait time prior to triage is not recorded (28). Additionally, there is a variance in how hospitals record this data. Patients may be registered in the treatment area while others are recorded even before triage. As we cannot accurately account for this variance in the NHAMCS datasets, our findings may be skewed. We removed all records with missing wait times which may incur concerns of missing data bias.

Race and ethnicity are sometimes not reported directly by the patient, but rather recorded by ED administrative personnel, who may do it based on perception. Studies of data from the Department of Veterans Affairs have shown that estimations of race and ethnicity based on perception are not accurate (31). This inexactness can also potentially skew our results.

Some of these differences that we are noting may be due to known differences and discontinuities between ICD-9CM coded data (years 2012-2015) and ICD-10-CM (years 2016-2018) coded data. Additionally, data from 2016 may reflect a transition period for hospitals. 


\section{Conclusion}

This study analyzed racial and ethnic disparities in ED wait time and LOV for MH/SU disorder patients using the 2012-2018 NHAMCS datasets. We examined these outcomes across all age groups based on their sociodemographic characteristics. Our findings suggest there are significant racial disparities in both ED wait time and LOV. Non-Hispanic Black patients experienced longer wait times, and non-Hispanic Black and Hispanic patients experienced longer LOV as compared to non-Hispanic White patients. Our findings also suggest significant differences based on insurance type, resulting in longer ED wait times for self-pay patients, and longer ED LOV for Medicaid or CHIP, Medicare, and unknown or other patients as compared to private insurance patients. A significant decrease in median ED wait times for MH/SU patients was observed, along with an increase in both $\mathrm{MH} / \mathrm{SU}$ disorder and general ED admission rates. These differences are a problem not only for patients and health systems but for the larger public health in the U.S. as they are related to population health and negatively affect the overall health of the nation. Additionally, they are a financial burden due both to increased medical care and loss of productivity. In conclusion, while several improvements were observed in our analysis, the trend of racial/ethnic and socio-economic disparities across the country's healthcare system needs further attention.

\section{List of Abbreviations}

MH/SU - Mental health or Substance use (SUD - substance use disorder)

NHAMCS - National hospital ambulatory medical care survey

CDC - Centers for disease control and prevention

ED - Emergency department

LOV - Length of visit

ICD-9-CM - International classification of diseases, ninth revision, clinical modification

POCT - Point-of-care testing

\section{Declarations}

\section{Ethics approval and consent to participate}

Not Applicable

\section{Consent for publication}

Not Applicable

\section{Availability of data and materials}

All data used are publicly available at the NHAMCS website:

https://www.cdc.gov/nchs/ahcd/datasets_documentation_related.htm

\section{Competing interests}

There are no competing interests

\section{Funding}

This study was funded in part by NIH grants R25MD011713, R01MH119177, and R01MH121907. Each of these 
grants are supporting research grants.

\section{Author's contributions}

JW is the corresponding author. She was involved at all levels of this paper from conceptualization and methodology, to writing the software and data curation along with writing and editing.

PG was involved in the initial coneptualization, methodology, software and writing of the original draft.

JA was involved in review and editing and validation.

SM was involved in review and editing and validation

JP was involved in the intiial conceptualization, methodology supervision.

All authors read and approved the final manuscript.

\section{Acknowledgements}

Not Applicable

\section{REFERENCES}

1. Stockdale SE, Lagomasino IT, Siddique J, McGuire T, Miranda J. Racial and ethnic disparities in detection and treatment of depression and anxiety among psychiatric and primary health care visits, 1995-2005. Med Care. 2008 Jul;46(7):668-77.

2. Williams DR, Priest N, Anderson N. Understanding Associations between Race, Socioeconomic Status and Health: Patterns and Prospects. Health Psychol Off J Div Health Psychol Am Psychol Assoc. 2016 Apr;35(4):407-11.

3. Kindig D, Stoddart G. What Is Population Health? Am J Public Health. 2003 Mar;93(3):380-3.

4. Xin H. Editorial: Health Disparities_-An Important Public Health Policy Concern. Front Public Health [Internet]. 2017 May 3 [cited 2020 Dec 3];5. Available from: https://www.ncbi.nlm.nih.gov/pmc/articles/PMC5413494/

5. Kindig DA. Understanding Population Health Terminology. Milbank Q. 2007 Mar;85(1):139-61.

6. Silberberg M, Martinez-Bianchi V, Lyn MJ. What Is Population Health? Prim Care. 2019 Dec;46(4):475-84.

7. National Academies of Sciences E, Division H and M, Practice B on PH and PH, States C on C-BS to PHE in the U, Baciu A, Negussie Y, et al. The State of Health Disparities in the United States [Internet]. Communities in Action: Pathways to Health Equity. National Academies Press (US); 2017 [cited 2020 Dec 3]. Available from: https://www.ncbi.nlm.nih.gov/books/NBK425844/

8. Snyder CR, Frogner BK, Skillman SM. Facilitating Racial and Ethnic Diversity in the Health Workforce. J Allied Health. 2018;47(1):58-65.

9. Kennedy J, Rhodes K, Walls CA, Asplin BR. Access to emergency care: restricted by long waiting times and cost and coverage concerns. Ann Emerg Med. 2004 May;43(5):567-73. 
10. Pines JM, Pollack CV, Diercks DB, Chang AM, Shofer FS, Hollander JE. The association between emergency department crowding and adverse cardiovascular outcomes in patients with chest pain. Acad Emerg Med Off J Soc Acad Emerg Med. 2009 Jul;16(7):617-25.

11. Seymour CW, Gesten F, Prescott HC, Friedrich ME, Iwashyna TJ, Phillips GS, et al. Time to Treatment and Mortality during Mandated Emergency Care for Sepsis. N Engl J Med. 2017 Jun 8;376(23):2235-44.

12. Sonis JD, Aaronson EL, Lee RY, Philpotts LL, White BA. Emergency Department Patient Experience: A Systematic Review of the Literature. J Patient Exp. 2018 Jun;5(2):101-6.

13. Pearlmutter MD, Dwyer KH, Burke LG, Rathlev N, Maranda L, Volturo G. Analysis of Emergency Department Length of Stay for Mental Health Patients at Ten Massachusetts Emergency Departments. Ann Emerg Med. 2017 Aug;70(2):193-202.e16.

14. Skinner HG, Blanchard J, Elixhauser A. Trends in Emergency Department Visits, 2006-2011: Statistical Brief \#179. In: Healthcare Cost and Utilization Project (HCUP) Statistical Briefs [Internet]. Rockville (MD): Agency for Healthcare Research and Quality (US); 2006 [cited 2021 Apr 21]. Available from: http://www.ncbi.nlm.nih.gov/books/NBK254201/

15. Delia D, Cantor JC. Emergency department utilization and capacity. Synth Proj Res Synth Rep. 2009 Jul;(17).

16. Newton MF, Keirns CC, Cunningham R, Hayward RA, Stanley R. Uninsured adults presenting to US emergency departments: assumptions vs data. JAMA. 2008 Oct 22;300(16):1914-24.

17. Kne T, Young R, Spillane L. Frequent ED users: patterns of use over time. Am J Emerg Med. 1998 Nov;16(7):648-52.

18. Rask KJ, Williams MV, McNagny SE, Parker RM, Baker DW. Ambulatory Health Care Use by Patients in a Public Hospital Emergency Department. J Gen Intern Med. 1998 Sep;13(9):614-20.

19. Appel G, Zaidi SR, Han BH, Avery JJ, Avery JD. A Call for Increased Psychiatric Training in Emergency Medicine: Physician Attitudes Toward Substance Use Disorders and Co-Occurring Borderline Personality Disorder. Prim Care Companion CNS Disord. 2020 Aug 13;22(4).

20. Hong V. Borderline Personality Disorder in the Emergency Department: Good Psychiatric Management. Harv Rev Psychiatry. 2016 Oct;24(5):357-66.

21. Dydyk AM, Jain NK, Gupta M. Opioid Use Disorder. In: StatPearls [Internet]. Treasure Island (FL): StatPearls Publishing; 2021 [cited 2021 Apr 21]. Available from: http://www.ncbi.nlm.nih.gov/books/NBK553166/

22. James K, Jordan A. The Opioid Crisis in Black Communities. J Law Med Ethics J Am Soc Law Med Ethics. 2018 Jun;46(2):404-21.

23. Alexander MJ, Kiang MV, Barbieri M. Trends in Black and White Opioid Mortality in the United States, 1979-2015. Epidemiology. 2018 Sep;29(5):707-15.

24. Pletcher MJ, Kertesz SG, Kohn MA, Gonzales R. Trends in Opioid Prescribing by Race/Ethnicity for Patients Seeking Care in US Emergency Departments. JAMA [Internet]. 2008 Jan 2 [cited 2021 Apr 21];299(1). Available from: http://jama.jamanetwork.com/article.aspx?doi=10.1001/jama.2007.64

25. Lu FQ, Hanchate AD, Paasche-Orlow MK. Racial/ethnic disparities in emergency department wait times in the United States, 2013-2017. Am J Emerg Med. 2021 Sep;47:138-44. 
26. Wu BU, Banks PA, Conwell DL. Disparities in emergency department wait times for acute gastrointestinal illnesses: results from the National Hospital Ambulatory Medical Care Survey, 1997-2006. Am J

Gastroenterol. 2009 Jul;104(7):1668-73.

27. Opoku ST, Apenteng BA, Akowuah EA, Bhuyan S. Disparities in Emergency Department Wait Time Among Patients with Mental Health and Substance-Related Disorders. J Behav Health Serv Res. 2018;45(2):204-18.

28. Houston C, Sanchez LD, Fischer C, Volz K, Wolfe R. Waiting for Triage: Unmeasured Time in Patient Flow. West J Emerg Med. 2015 Jan;16(1):39-42.

29. Sealy-Jefferson S, Vickers J, Elam A, Wilson MR. Racial and Ethnic Health Disparities and the Affordable Care Act: a Status Update. J Racial Ethn Health Disparities. 2015 Dec;2(4):583-8.

30. Institute of Medicine (US) Committee on Quality of Health Care in America. Crossing the Quality Chasm: A New Health System for the 21st Century [Internet]. Washington (DC): National Academies Press (US); 2001 [cited 2021 Apr 29]. Available from: http://www.ncbi.nlm.nih.gov/books/NBK222274/

31. Kressin NR, Chang B-H, Hendricks A, Kazis LE. Agreement between administrative data and patients' selfreports of race/ethnicity. Am J Public Health. 2003 Oct;93(10):1734-9.

32. Butryn T, Bryant L, Marchionni C, Sholevar F. The shortage of psychiatrists and other mental health providers: Causes, current state, and potential solutions. Int J Acad Med. 2017;3(1):5-9.

33. Jarvis PRE. Improving emergency department patient flow. Clin Exp Emerg Med. 2016 Jun 30;3(2):63-8.

34. Hsu H, Greenwald PW, Clark S, Gogia K, Laghezza MR, Hafeez B, et al. Telemedicine Evaluations for LowAcuity Patients Presenting to the Emergency Department: Implications for Safety and Patient Satisfaction. Telemed J E-Health Off J Am Telemed Assoc. 2020 Aug;26(8):1010-5.

35. Rooney KD, Schilling UM. Point-of-care testing in the overcrowded emergency department - can it make a difference? Crit Care. 2014 Dec 8;18(6):692.

36. Grover CA, Sughair J, Stoopes S, Guillen F, Tellez L, Wilson TM, et al. Case Management Reduces Length of Stay, Charges, and Testing in Emergency Department Frequent Users. West J Emerg Med. 2018 Mar;19(2):238-44.

37. Kaplan G, Lopez MH, McGinnis JM, Care C on OS in H, Medicine I of. Issues in Access, Scheduling, and Wait Times [Internet]. Transforming Health Care Scheduling and Access: Getting to Now. National Academies Press (US); 2015 [cited 2020 Dec 3]. Available from: https://www.ncbi.nlm.nih.gov/books/NBK316141/

38. van der Linden MC, Meester BEAM, van der Linden N. Emergency department crowding affects triage processes. Int Emerg Nurs. 2016 Nov;29:27-31.

39. Snowden LR, Catalano R, Shumway M. Disproportionate use of psychiatric emergency services by african americans. Psychiatr Serv Wash DC. 2009 Dec;60(12):1664-71.

40. Kim G, Dautovich N, Ford K-L, Jimenez DE, Cook B, Allman RM, et al. Geographic variation in mental health care disparities among racially/ethnically diverse adults with psychiatric disorders. Soc Psychiatry Psychiatr Epidemiol. 2017;52(8):939-48.

41. Mays VM, Jones A, Delany-Brumsey A, Coles C, Cochran SD. Perceived Discrimination in Healthcare and Mental Health/Substance Abuse Treatment Among Blacks, Latinos, and Whites. Med Care. 2017 Feb;55(2):173-81. 
42. James CA, Bourgeois FT, Shannon MW. Association of race/ethnicity with emergency department wait times. Pediatrics. 2005 Mar;115(3):e310-315. 

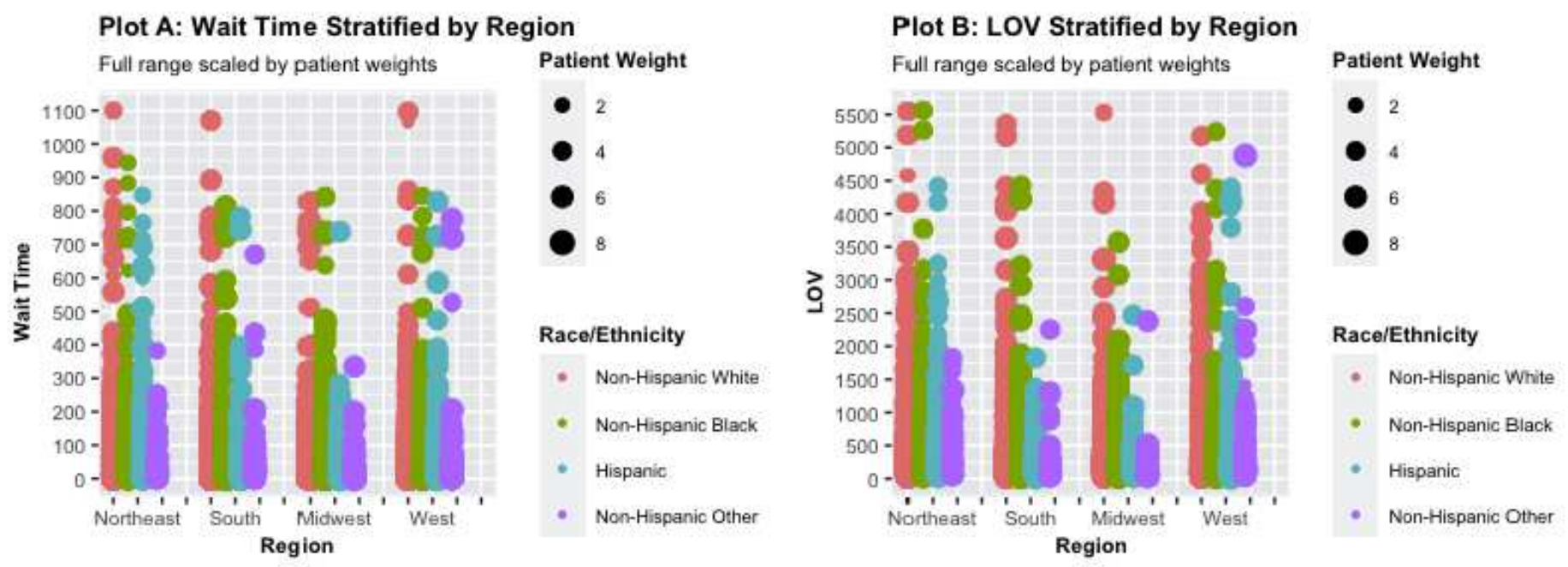

Plot C: Wait Time Stratified by Region

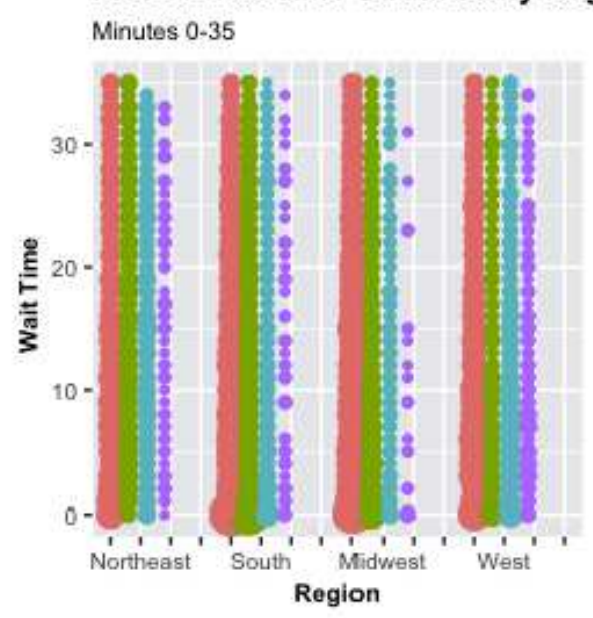

\section{Count}

Plot D: LOV Stratified by Region

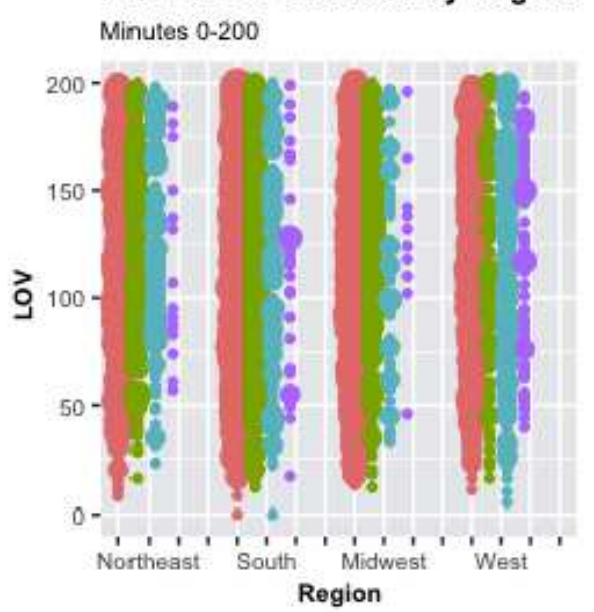

\section{Race/Ethnicity}

- Non-Hispanic White

- Non-Hispanic Black

- Hispanio

- Non-Hispanic Other

\section{Figure 1}

Wait time and LOV stratified by region. In plots $A$ and B each point represents a unique ED visit, with the size of the point informing the patient weight for that visit. The four colors inform the race/ethnicity of the patient. In plots $C$ and $D$, each point represents a count for the number of unique patients. Additionally, these two plots detail the lower range of wait time and LOV minutes. 


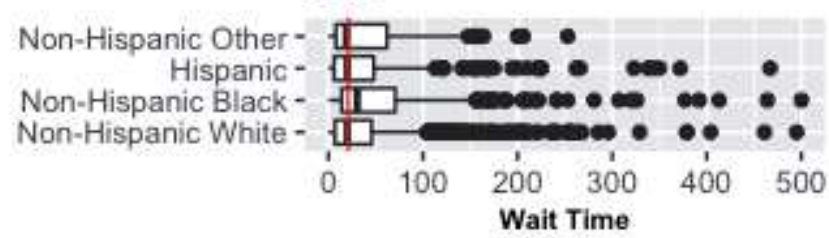

2013

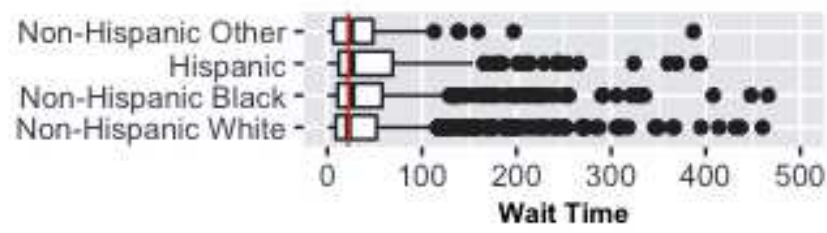

2014

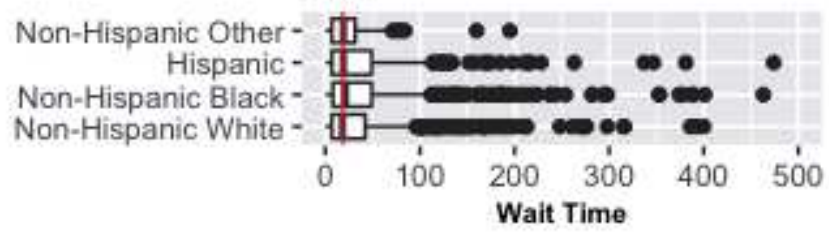

2015

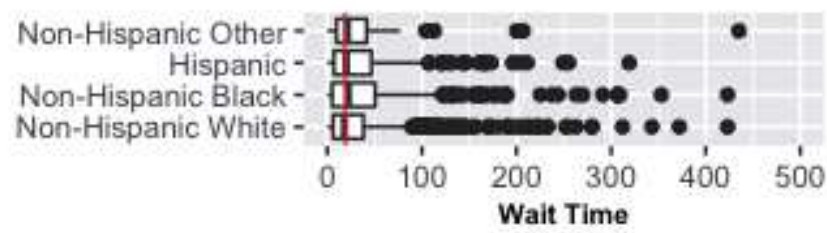

2016

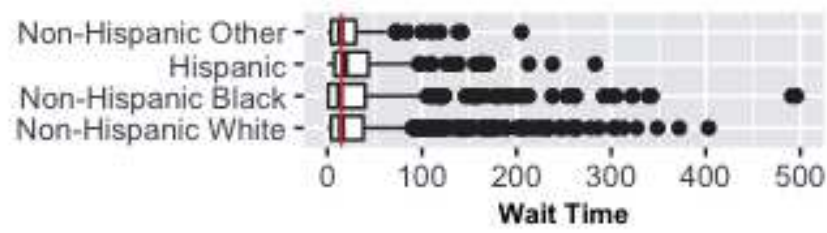

2018

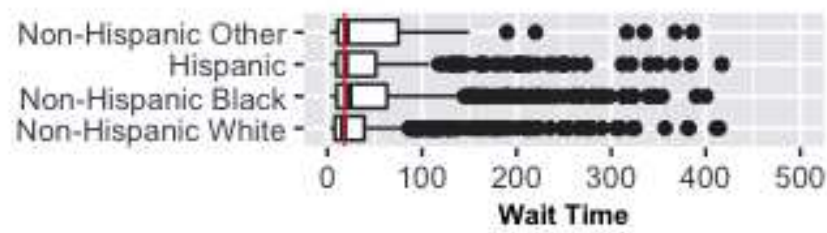

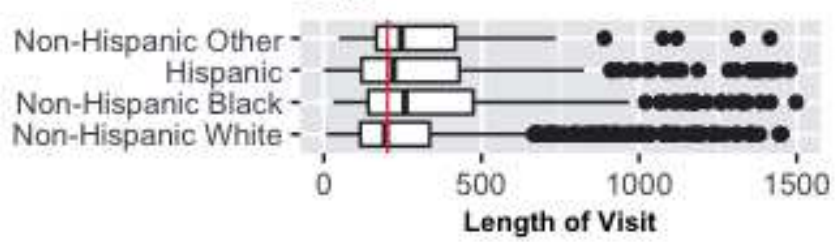

2013

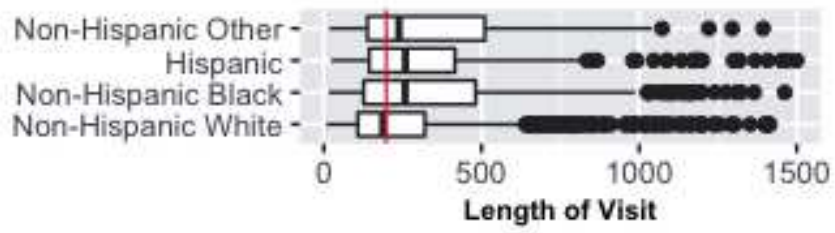

2014

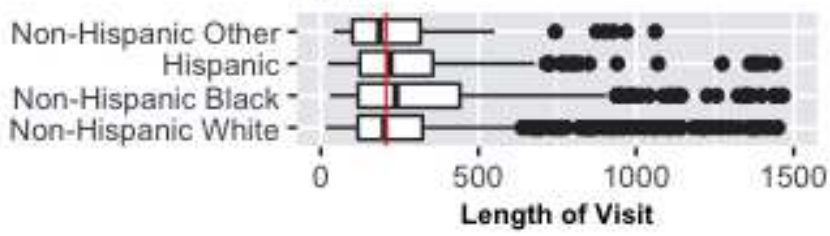

\section{5}

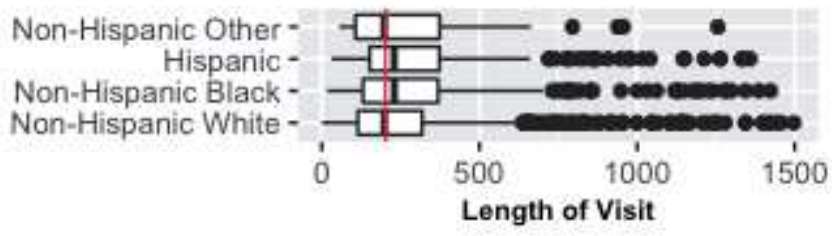

2017

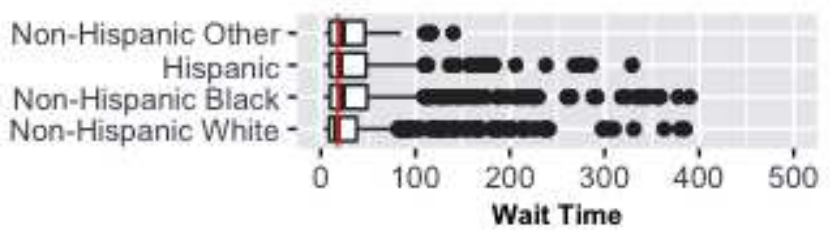

2018

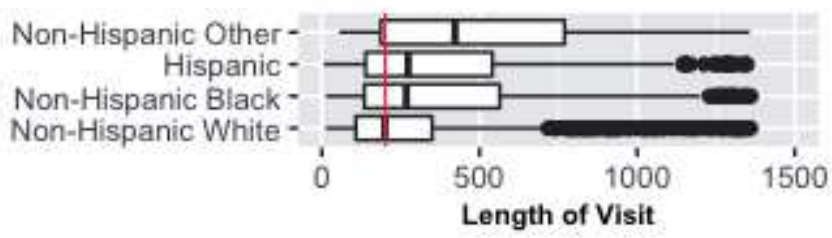

\section{Figure 2}

Median and quartile ranges of the wait time/ LOV for each racial/ethnic group year by year. The red line shows comparative medians using NHW as reference. NOTE: LOV data was not available for the years 2016-2017. 

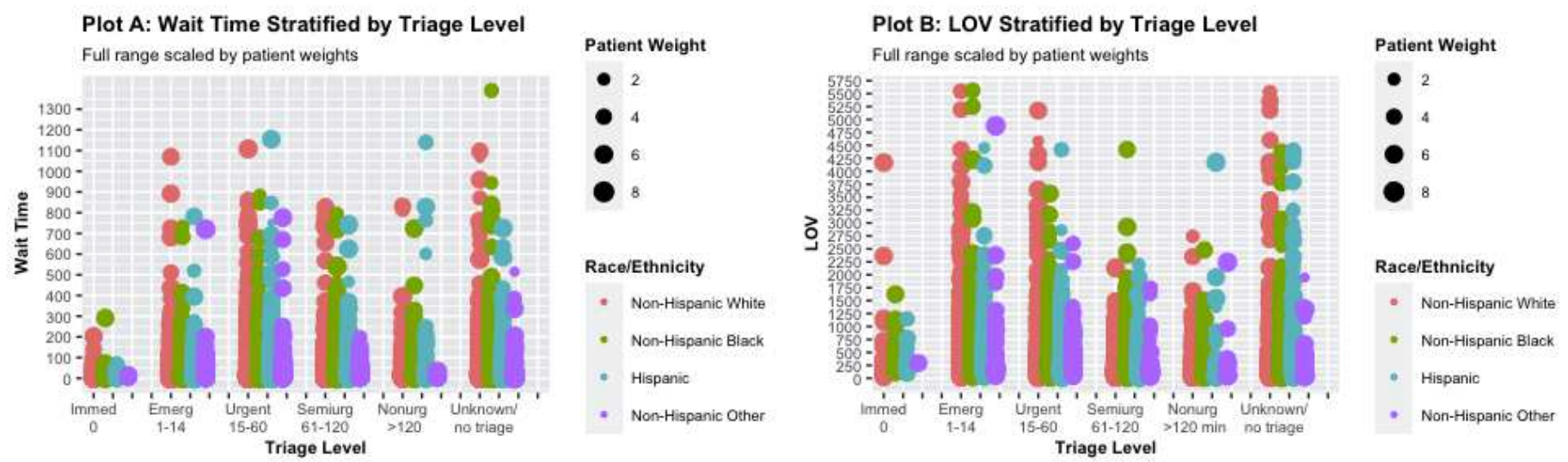

Plot C: Wait Time Stratified by Triage Level
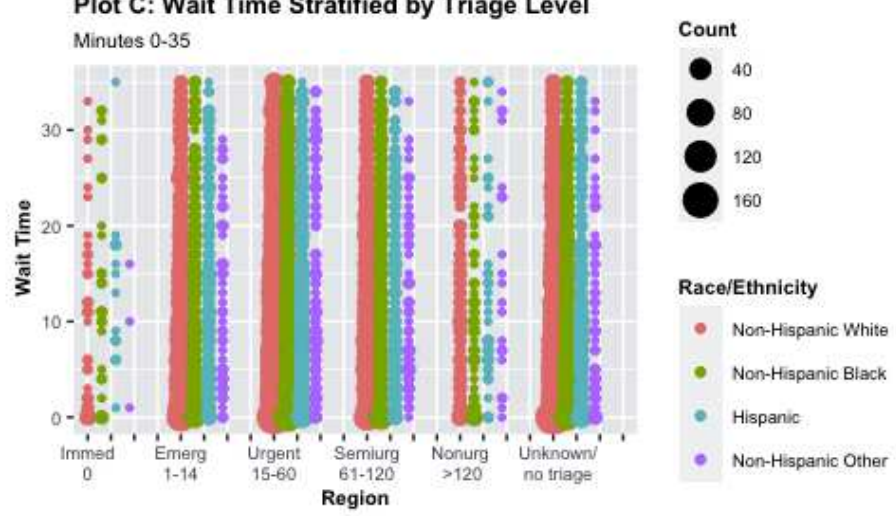

Plot D: LOV Time Stratified by Triage Level Minutes 0-300

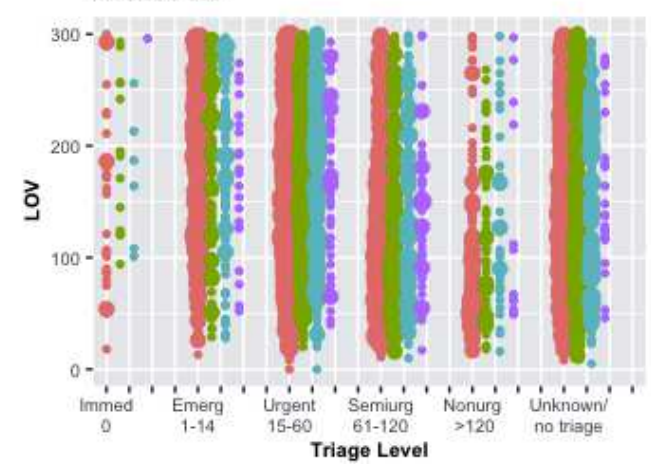

Count
10

Race/Ethnicity

- Non-Hispanic White

- Non-Hispanic Black

- Hispanic

- Non-Hispanic Other

\section{Figure 3}

Wait time and LOV stratified by triage level. In plots $A$ and $B$ each point represents a unique ED visit, with the size of the point informing the patient weight for that visit. The four colors inform the race/ethnicity of the patient. In plots $C$ and $D$, each point represents a count for the number of unique patients. Additionally, these two plots detail the lower range of wait time minutes.

\section{Supplementary Files}

This is a list of supplementary files associated with this preprint. Click to download.

- SUTPPNHAMCSSupplementalFiles.pdf 